\title{
Lieu du décès au Burkina : influence des caractéristiques sociodémographiques et environnementales
}

\author{
Aristide Romaric Bado ${ }^{1 a, c}$, Séni Kouanda ${ }^{a, b}$, Slim Haddad ${ }^{d}$ \\ a Institut de Recherche en Sciences de la Santé (IRSS) / Centre National de la Recherche \\ Scientifique et Technologique (CNRST), Ouagadougou, Burkina Faso \\ b Institut Africain de santé publique (IASP) \\ c Department of Statistics and Population Studies, University of the Western Cape \\ ¿ Département de médecine sociale et préventive, Faculté de Médecine, Université Laval, \\ Québec, Canada
}

\begin{abstract}
This paper explores the factors associated with the place of death in Burkina Faso, based on mortality data from the Kaya Health and Demographic Surveillance System (Kaya HDSS). A multilevel logistic regression model with random intercept is used to determine the factors associated with the place of death. More than half of the deaths $(55 \%)$ occur at home. Age, place of residence, distance to the health centre and cause of death are statistically associated with the place of death. Seniors $(50$ and over) are more likely to die at home compared to other age groups $(66.8 \mathrm{I} \%$ against $35.9 \%$ for $5-\mathrm{I} 4$ years and $44.9 \%$ among children under 5 years, $p=0.00 \mathrm{I}$ ). The multivariate results confirm the effect of age, place of residence, living standards quintile and cause of death. The high proportion of deaths occurring at home challenges policy makers in the health system and calls for programs to adapt the supply of health care.
\end{abstract}

Keywords: Place of death; determinants; cause of death; mortality; Burkina Faso

\section{Résumé}

Cet article explore les facteurs associés au lieu de décès au Burkina Faso à partir des données de mortalité du système de surveillance démographique et de santé de Kaya (Kaya HDSS). Un modèle de régression logistique multiniveau à intercept aléatoire est utilisé pour déterminer les facteurs associés au lieu de décès. Plus de la moitié des décès (55\%) surviennent à domicile. En analyse bivariée, l'âge, le milieu de résidence, la distance par rapport au centre de santé et la cause de décès sont statistiquement associées au lieu de décès. Les personnes âgées $(50$ ans et +$)$ sont plus nombreux à décéder à domicile par rapport aux autres groupes d'âges $(66,81 \%$ contre $35,9 \%$ chez les $5-14$ ans et $44,9 \%$ chez les moins de 5 ans; $p=0,00 \mathrm{I}$ ). Les résultats multivariés confirment l'effet de l'âge, du milieu de résidence, du quintile de niveau de vie et de la cause de décès. La forte proportion des décès à domicile interpelle les décideurs du système de santé et incite à développer davantage de programmes visant à adapter l'offre des soins aux besoins des populations.

Mots-clés: Lieu du décès; déterminants; cause de décès; mortalité; Burkina Faso.

\footnotetext{
I Corresponding author: Aristide Romaric Bado Institut de Recherche en Sciences de la Santé (IRSS) et Centre National de la Recherche Scientifique et Technologique (CNRST), Ouagadougou, Burkina Faso

03 BP 7| 92 Ouagadougou 03 Burkina Faso

Email: arbado@irss.bf

Tel: +22650333594
} 


\section{Introduction}

La connaissance des facteurs qui influencent le lieu de décès est important pour la planification en santé publique, l'allocation des ressources et la mesure de l'impact des interventions. Les décès qui surviennent à domicile de maladies infectieuses et parasitaire, de causes périnatales et de maladies liées à une carence nutritionnelle sont a priori évitables par un recours aux centres de santé où une prise en charge rapide et efficace est possible (Haddad 20l0). Mourir jeune, ou pendant l'accouchement ou en suite de couches à domicile peut souvent s'expliquer par le manque d'accessibilité des services de santé modernes. Selon Cohen J. et al. (2007), il est important pour la politique de santé publique d'étudier les préférences des individus en matière de lieu du décès, d'acquérir une meilleure compréhension des facteurs qui conditionnent le lieu du décès, et de déterminer les profils des personnes qui meurent dans un centre de santé ou à domicile. Ces informations sont utiles à l'élaboration et la mise en œuvre de programmes de soins palliatifs de qualité qui doivent permettre aux individus de mourir dans des conditions dignes (Cárdenas-Turanzas et al. 20ll). Dans les pays comme le Burkina Faso, où l'accès aux soins reste problématique, l'analyse du lieu de décès pourrait permettre de renseigner sur les besoins non couverts en termes de prise en charge.

La littérature sur les facteurs influençant le lieu de décès dans les pays développés est abondante. Plusieurs de ces études ont été réalisées sur des personnes âgées (Klinkenberg et al. 2005; M. Lazenby et al. 2010; Ruiz-Ramos and Javier 201 I), les jeunes enfants (Craig and Mancini 20I2), les patients atteints de cancer (Gallo et al. 200I; Gomes and Higginson 2006; Jack and O'Brien 20I0; D. Munday et al. 2009; Neergaard et al. 2012; Wright et al. 20l0), et les personnes recevant des soins palliatifs en institution ou à domicile (McWhinney et al. 1995). Dans ces pays, l'intérêt est surtout porté sur les soins palliatifs dont l'objectif est de soulager les douleurs physiques et de préserver la qualité de vie du malade au cours de ces derniers moments de vie.

Dans les pays africains, très peu d'études se sont intéressées aux facteurs associés au lieu de décès (Anteneh et al. 2013; J. M. Lazenby and Olshvevski 2012). Une des rares études sur ce thème a été réalisée à Addis Abeba, à l'aide d'un recensement des décès lors d'inhumations dans les cimetières, tandis qu'une étude menée au Botswana a porté sur un échantillon des décès des personnes de 80 ans extraits du registre de l'état civil (Lazenby \& Olshvevski, 20I2). L'une des insuffisances de ces deux études études est d'avoir exclu les décès de moins de 15 ans quand on sait que les statistiques sur les décès des enfants et particulièrement ceux des enfants de moins de cinq constituent des indicateurs importants sur la santé des populations.

La plupart des études qui se sont focalisées sur le recours aux soins en Afrique sub-saharienne mettent en évidence de profondes barrières socio-culturelles (Beninguisse and Fournier 2003) et économiques (Wagstaff 2002) ou géographiques limitant l'accès aux soins. Le lieu de décès et les facteurs qui les influencent apparaissent comme secondaires et ont été peu étudiés (M. Lazenby et al. 20l0).

L'objectif de cette étude est d'identifier les facteurs qui influencent le lieu de décès (à domicile ou au sein d'un centre de santé) en explorant l'effet des variables individuelles et contextuelles sur le fait de décéder à la maison à partir de données collectées dans un site démographique centré sur une ville moyenne au Burkina Faso.

\section{Revue de littérature}

Dans les pays développés, les études ont mis en exergue plusieurs facteurs prédictifs de lieu de décès. Gomes et al. (2006) ont proposé un cadre conceptuel des facteurs associés au lieu de décès qui a ensuite été utilisé par d'autres pour comprendre notamment les déterminants du lieu de décès chez les patients souffrant de cancer (D. Munday et al. 2009; Daniel Munday et al. 2007; Neergaard et al. 2012). Ce modèle conceptuel classifie les facteurs selon :

I) les facteurs sociodémographiques (le sexe, l'âge, l'ethnie, la préférence individuelle pour un décès à domicile ou non, le niveau d'instruction, le statut matrimonial...)

2) les facteurs liés à la maladie (le type de maladie, les symptômes)

3) les facteurs liés à l'environnement (l'utilisation des services de santé, le soutien social disponible).

Les facteurs sociodémographiques influencent principalement la prise de décision sur l'opportunité de demander des soins en cas de maladie, et ceci a été observé aussi bien dans les pays développés (Gallo et al. 200I; Gomes and Higginson 2006) que ceux en développement (Anteneh et al. 2013; J. M. Lazenby and Olshvevski 20l2). L'âge est souvent présenté comme un indicateur d'accumulation d'expérience (Gabrysch and Campbell 2009) qui a des implications sur la préférence aux soins de santé (modernes ou traditionnels), ainsi que sur la perception de la maladie et de la mort. Le décès d'une personne âgée est plus facilement accepté que celle d'une personne jeune (Grunier et al. 2007), et donc davantage susceptible de survenir sans 
assistance médicale. Antenet et al.(20/3) ont ainsi montré qu'en Ethiopie, la probabilité de décéder dans un centre de santé plutôt qu'à domicile diminue avec l'âge.

La religion et l'ethnie comptent également parmi les principaux déterminants du recours aux soins (Gabrysch and Campbell 2009), car ils influencent les croyances sur les maladies et la mort ainsi que la perception des individus des services de santé et donc du lieu de décès.

D'autres variables individuelles, telles que le statut matrimonial (McWhinney et al. 1995, 2004), le sexe (Ali et al. 20II; Anteneh et al. 2013; Baider and Surbone 2007; Beng et al. 2009; Brazil et al. 2002; Ikezaki and Ikegami 20l I; M. Lazenby et al. 2010; Ruiz-Ramos and Javier 20I I), la profession (Anteneh et al. 20/3) et le niveau d'instruction (Anteneh et al. 2013; Gallo et al. 200I; Tang et al. 2010) ont aussi été pris en compte dans les études comme étant des facteurs potentiels du lieu de décès.

Selon les cadre conceptuels de Gomes et Higginson (2006) et de Gruneir et al.,(2007) les facteurs liés à la maladie sont susceptibles d'influencer le lieu de décès. Les décès dus à des maladies de courte durée telles que l'infarctus du myocarde, peuvent être plus susceptibles de survenir à domicile alors que les patients atteints de maladies chroniques sont plus susceptibles de décéder dans un hôpital (Grunier et al. 2007). Toutefois, dans une situation de manque d'infrastructures hospitalières (insuffisance de chambres et de lits d'hospitalisation) comme le cas du Burkina Faso, on s'attend à ce que davantage de personnes souffrant de maladies chroniques ou ayant atteint des stades terminaux meurent à domicile. En effet, dans un contexte de moyens limités, la priorité pour l'occupation des lits serait davantage accordée aux nouveaux malades, souffrant de problèmes de santé aigus. Par ailleurs, les maladies chroniques engendrent souvent des dépenses catastrophiques de santé que les ménages ne peuvent plus supporter, ce qui se solde par un renoncement aux soins en milieu hospitalier. En cas de paiement direct, le risque de dépenses catastrophiques est généralement plus grand pour les ménages qui comptent une personne âgée, handicapée ou atteinte d'une maladie chronique, à la fois parce qu'ils ont plus souvent recours aux services de santé et parce que leurs moyens financiers sont moindres (Ahishakiyé 2008; Su et al. 2006; Xu et al. 2003). En l'absence de mécanismes de protection efficaces, ces ménages sont constamment exposés au double risque de maladie et de difficultés financières (Xu et al. 2003).

La littérature sur le lieu de décès pointe du doigt l'influence importante des facteurs liés à l'environnement tels que le milieu de résidence, la présence et l'accessibilité de centres de santé, la capacité d'accueil de ces centres, ainsi que le soutien social dont a pu bénéficier la personne décédée. Gomes \& Higginson (2006) ont montré qu'en Europe, le fait de résider en milieu rural accroit la propension à mourir hors d'un centre de santé. De même, les résultats des études empiriques, en Italie (Beccaro et al. 2006; Costantini et al. 2000), en France (Chvetzoff et al. 2005) et dans plusieurs pays d'Europe (Cohen et al. 2010; Higginson et al. 1999) ont confirmé l'existence d'une association significative entre le milieu de résidence et le lieu des décès. En Italie, les proportions des décès à domicile étaient de $60,4 \%, 53,4 \%$ et $43,2 \%$ respectivement en milieu semi-urbain, rural et urbain (Costantini et al. 2000). Ces proportions étaient par ailleurs plus élevées au sud du pays, comparativement aux autres régions (Beccaro et al. 2006). Dans les pays à faibles revenus comme le Burkina Faso, de grandes disparités d'allocation des ressources et des infrastructures socio-sanitaires existent entre régions et milieu de résidence. Dans les zones rurales en général, les services et les infrastructures de santé sont rares ou de mauvaise qualité et cela constitue une barrière à l'utilisation des soins de santé, qui peut parfois se combiner à l'effet des croyances traditionnelles défavorables au recours aux soins modernes. Au Burkina, plus de moitié de l'ensemble du personnel de santé est concentrée dans les deux grandes villes (Ouagadougou et Bobo-Dioulasso) (Seni Kouanda et al. 20 I4). Dans ces conditions, mourir à domicile sera nettement plus fréquent au milieu rural.

\section{Données et Méthodes Cadre de l'étude}

L'étude se déroule sur le site de surveillance démographique et épidémiologique de Kaya mis en place en fin 2007 par l'Equipe de recherche interdisciplinaire sur le $\mathrm{VIH} / \mathrm{Sida}$ et la santé de la reproduction (ERIS) de l'Institut de recherche en sciences de la santé (IRSS). Le site de l'étude est situé dans le district sanitaire de Kaya dans la province du Sanmatenga, dans la région du Centre-Nord du Burkina Faso. II couvre la ville de Kaya ainsi que 18 villages qui l'entourent. La population résidente comptait environ 67000 habitants en début d'année 2012 , et cette population est considérée comme étant à $70 \%$ urbaine et à $30 \%$ rurale (Séni Kouanda et al. 20।3).

\section{Sources des données et échantillon de l'étude}

Les données utilisées proviennent des registres de décès du Kaya HDSS couvrant la période de janvier 2008 à décembre 2010. Les causes de décès ont été obtenues par la méthode des autopsies verbales 
réalisée pour chaque décès notifié au sein du site de l'observatoire à l'aide du questionnaire standardisé de I'OMS (WHO 2009). Les questionnaires sont ensuite été analysés par des médecins codeurs qui identifient la cause probable de décès. Les causes de décès sont par la suite codées selon $10 \mathrm{e}$ révision de la classification internationale des maladies (CIM-I0).

Au total, 603 décès, tous âges confondus, ont été enregistrés dans la population couverte par le système de surveillance pendant la période de l'étude. L'analyse porte sur 596 décès (soit $98,8 \%$ de l'ensemble des décès) pour lesquels des informations sur les causes de décès sont disponibles.

\section{Variables de l'étude}

Notre variable dépendante principale est simplement l'occurrence d'un décès à domicile plutôt que dans une formation sanitaire. Ces formations sanitaires incluent tout type de structures, qu'elles soient publics ou privées, de première ligne ou de référence.

Parmi les variables indépendantes, on distingue d'abord les facteurs sociodémographiques, qui comprennent le sexe, l'âge, le lien de parenté avec le chef de ménage, l'ethnie, la religion et le niveau d'instruction. Les facteurs environnementaux sont au nombre de trois: le milieu de résidence, la distance séparant le ménage du centre de santé le plus proche, et le quintile du niveau du ménage. Cette dernière variable a été construite à partir des biens de possessions et des biens durables du ménage. Enfin, la cause de la maladie sera la seule variable prise en compte dans nos analyses qui traduit les facteurs liés à la maladie. En effet, les variables (durée de la maladie, le type de recours aux soins et la chronicité de la maladie) ne figuraient pas dans nos bases de données.

\section{Méthodes d'analyse}

Des premières analyses permettent d'examiner le degré d'association entre les variables explicatives et la variable dépendante à l'aide d'un test de Chi ${ }^{2}$. Elles sont suivies d'une régression logistique multiniveau multiple. Cette méthode d'analyse a été utilisée pour évaluer l'effet des variables individuelles et environnementales sur le lieu de décès tout en tenant compte de la nature hiérarchique des données.
L'interprétation se fonde sur l'examen des ratios de côtes (odd ratio (OR)). Un modèle multiniveau de régression logistique à intercept aléatoire a été utilisé et nous avons implémenté 4 modèles statistiques. Le modèle nul (Modèle 0 ) sans l'introduction de variables indépendantes permet d'abord d'évaluer l'influence des caractéristiques non observées sur la variation globale du lieu de décès. Ensuite, trois modèles de régression logistique à valeur aléatoire à deux niveaux ont été ajustés pour estimer les associations entre les déterminants potentiels et la probabilité de décéder à domicile. Le premier modèle inclus les caractéristiques individuelles, le second prend en compte les variables liés à l'environnement et le troisième modèle inclut les caractéristiques individuelles, environnementales et la cause de décès. Par souci de parcimonie, seuls les résultats du modèle complet sont présentés. Toutes les analyses statistiques ont été réalisées avec l'aide STATA 12.1 et la procédure xtmelogit.

\section{Résultats}

\section{Analyses descriptives}

La première colonne du tableau I présente les caractéristiques sociodémographiques des personnes décédées. Parmi les 596 décès, 328 (55\%) ont eu lieu à domicile. L'échantillon se caractérise par une prédominance de personnes du milieu urbain $(58,4 \%)$ et vivant à moins de 5 kilomètres $(\mathrm{kms})$ d'une formation sanitaire $(80,2 \%)$. La majorité des personnes décédées sont musulmans $(73 \%)$ et appartiennent à l'ethnie Mossi (87\%). Les personnes ayant fréquenté l'école sont faiblement représentées $(\mathrm{II} \%)$ et celles exerçant dans l'agriculture représentent $39 \%$ des décès. Le paludisme est la principale cause de décès (16\%) dans l'ensemble. II est suivi des causes périnatales (11\%), des autres maladies infectieuses (8.6\%), des maladies de l'appareil circulatoire (dont 29 décès sur 48 causés par l'hypertension artérielle) (8.1\%), des bronchopneumo/pneumopathies (7.2\%) et des causes indéterminées (8\%). Les affections rénales et urologiques représentent quant à elles $3,4 \%$ des décès, dont un peu moins de la moitié sont causés par des insuffisances rénales chroniques 
Tableau I : Description de l'échantillon

\begin{tabular}{|c|c|c|c|c|c|c|}
\hline \multirow{3}{*}{$\begin{array}{l}\text { Variables } \\
\end{array}$} & \multirow{3}{*}{ Modalités } & \multirow{2}{*}{\multicolumn{2}{|c|}{ Description de l'échantillon }} & \multicolumn{3}{|c|}{ Lieu de décès } \\
\hline & & & & \multirow{2}{*}{$\begin{array}{l}\begin{array}{l}\text { Domicile } \\
(\mathrm{n}=328)\end{array} \\
\% \\
\end{array}$} & \multirow{2}{*}{$\begin{array}{l}\text { Hôpital } \\
(n=268)\end{array}$} & \multirow[t]{2}{*}{$\begin{array}{l}\text { Significativité } \\
\text { du test de } \\
\text { chi2 }\end{array}$} \\
\hline & & $\%$ & $(n=596)$ & & & \\
\hline \multirow{2}{*}{ Sexe } & Masculin & 45,5 & 271 & 55,9 & $44, I$ & \multirow{2}{*}{$p=0,703$} \\
\hline & Féminin & 54,4 & 324 & 54,3 & 45,7 & \\
\hline \multirow{3}{*}{ Age } & $0-14$ & 29,7 & 177 & 42,9 & 57,1 & $p=0,00 I$ \\
\hline & $15-59$ & 33,4 & 199 & 45,7 & 54,3 & \\
\hline & $60+$ & 36,9 & 220 & 73,2 & 26,8 & \\
\hline \multirow{3}{*}{ Religion } & Musulmane & 73,7 & 439 & 54,2 & 45,8 & $p=0,798$ \\
\hline & Catholique & 17,3 & 103 & 57,3 & 42,7 & \\
\hline & Autre & 9,1 & 54 & 57,4 & 42,6 & \\
\hline \multirow{2}{*}{ Ethnie } & Mossi & 87,2 & 520 & 55,2 & 44,8 & $p=0,839$ \\
\hline & Autre & 12,8 & 76 & 53,9 & $46, I$ & \\
\hline \multirow{2}{*}{$\begin{array}{l}\text { Avoir été à } \\
\text { l'école }\end{array}$} & Non & 88,9 & 530 & 57,0 & 43,0 & $p=0,007$ \\
\hline & Oui & $\mathrm{II}, \mathrm{I}$ & 66 & 39,4 & 60,6 & \\
\hline \multirow{2}{*}{ Profession } & Agriculteurs & 38,8 & 231 & 54,8 & 45,2 & $p=0,883$ \\
\hline & Non agriculteurs & 61,2 & 365 & 55,4 & 44,6 & \\
\hline \multirow{3}{*}{$\begin{array}{l}\text { Statut } \\
\text { matrimonial }\end{array}$} & Célibataire & 39,4 & 235 & 44,7 & 55,3 & $p=00 I$ \\
\hline & Marié & 43,3 & 258 & 55,8 & 44,2 & \\
\hline & Séparé/divorcé & 17,3 & 103 & 76,7 & 23,3 & \\
\hline \multirow{3}{*}{ Année de décès } & 2008 & 30,9 & 184 & 57.1 & 42.9 & $p=0.506$ \\
\hline & 2009 & 33,2 & 198 & 56.6 & 43.4 & \\
\hline & 2010 & 35,9 & 214 & 51.9 & 48.1 & \\
\hline \multirow{2}{*}{$\begin{array}{ll}\begin{array}{l}\text { Milieu } \\
\text { résidence }\end{array} & \mathrm{de} \\
\end{array}$} & Urbain & 58,4 & 348 & 46,6 & 53,4 & \multirow{2}{*}{$\mathrm{p}<0,00 \mathrm{I}$} \\
\hline & Rural & 41,6 & 248 & 66,9 & 33,1 & \\
\hline \multirow{5}{*}{$\begin{array}{l}\text { Quintile de } \\
\text { niveau de vie }\end{array}$} & QI (Très pauvre) & 17.1 & 102 & 73,5 & 26,5 & \multirow{5}{*}{$p=0,001$} \\
\hline & Q2 & 18.5 & 110 & 58,2 & 41,8 & \\
\hline & Q3 & 22.5 & 134 & 52,2 & 47,8 & \\
\hline & Q4 & 26.5 & 158 & 50,6 & 49,4 & \\
\hline & Q5 (très nantis) & 15.4 & 92 & 42,4 & 57,6 & \\
\hline \multirow{3}{*}{$\begin{array}{lr}\text { Distance } & \text { par } \\
\text { rapport } & \text { au } \\
\text { centre de santé }\end{array}$} & $<5 \mathrm{~km}$ & 80,2 & 478 & 50,6 & 49,4 & \\
\hline & $5-9 \mathrm{~km}$ & 16,9 & 101 & 71,3 & 28,7 & $p<0,001$ \\
\hline & $10 \&+\mathrm{km}$ & 2,9 & 17 & 82,4 & 17,6 & \\
\hline & $\begin{array}{l}\text { Maladies infectieuses } \\
\text { et parasitaire (sans le } \\
\text { paludisme) }\end{array}$ & 4,2 & 25 & 64,0 & 36,0 & \\
\hline & Tumeurs & 6,3 & 38 & 60,5 & 39,5 & \\
\hline & $\begin{array}{l}\text { Troubles nutritionnels } \\
\text { et endocriniens }\end{array}$ & 5,0 & 30 & 43,3 & 56,7 & \\
\hline & $\begin{array}{l}\text { Maladies de l'appareil } \\
\text { circulatoire }\end{array}$ & 8,1 & 48 & 43,8 & 56,25 & \\
\hline & $\begin{array}{l}\text { Affections du tube } \\
\text { digestif }\end{array}$ & 4,2 & 25 & 52,0 & 48,0 & \\
\hline & $\begin{array}{l}\text { Affections rénales et } \\
\text { urologiques }\end{array}$ & 3,4 & 20 & 65,0 & 35,0 & \\
\hline Cause de décès & $\begin{array}{l}\text { Causes périnatales de } \\
\text { décès }\end{array}$ & $\mathrm{II}, \mathrm{I}$ & 66 & 50,0 & 50,0 & $p=0,019$ \\
\hline & Causes externes & 5,2 & 31 & 74,2 & 25,8 & \\
\hline & Indéterminé & 7,9 & 47 & 72,3 & 27,7 & \\
\hline & $\begin{array}{l}\text { Broncho } \\
\text { pneumo/pneumopathie }\end{array}$ & 7,2 & 43 & 67,4 & 32,6 & \\
\hline & $\begin{array}{ll}\begin{array}{l}\text { Diarrhée } \\
\text { infectieuse }\end{array} & \text { présumée } \\
\end{array}$ & 5,0 & 30 & 60,0 & 40,0 & \\
\hline & $\begin{array}{l}\text { Maladies infectieuses } \\
\text { non précise }\end{array}$ & 8,6 & 51 & 43,1 & 56,9 & \\
\hline & Méningite & 4,4 & 26 & 38,5 & 61,5 & \\
\hline & Paludisme & 15,8 & 94 & 53,2 & 46,8 & \\
\hline & Septicémie & 3,7 & 22 & 45,4 & 54,6 & \\
\hline & $\mathrm{N}$ & 100,0 & 596 & 55,1 & 44,9 & \\
\hline
\end{tabular}




\section{Analyse différentielle selon le lieu de décès}

II ressort des résultats de l'analyse différentielle du lieu de décès présentés dans les colonnes tableau I que l'âge, le milieu de résidence, le niveau de vie, la distance par rapport au centre de santé et la cause de décès sont statistiquement associés à l'occurrence d'un décès à domicile. Les personnes âgées de 50 ans et plus sont proportionnellement plus nombreuses à décéder à domicile que celles des autres groupes d'âges $(66,81 \%$ de décès à domicile parmi les 50 ans et plus comparativement à $35,9 \%$ chez les $5-14$ ans et $44,9 \%$ chez les moins de 5 ans; $p=0,00 \mathrm{I}$ ). Les proportions des décès survenus à domicile sont par ailleurs plus élevées en milieu rural $(66,9 \%)$ et parmi les personnes de quintile de niveau très pauvre (73,5\%). Plus de $65 \%$ des décès par causes externes (c'est-à-dire les décès par suite d'accident de la circulation, de blessures, de morsure de reptiles, ou par agression), par causes indéterminées, par broncho-pneumopathies et par affections rénales et urologiques ont eu lieu hors d'une structure de santé. La proportion de décès survenant à domicile est également supérieure à $60 \%$ pour les personnes ayant souffert de maladies diarrhéiques d'origine infectieuse, de tumeurs, maladies infectieuses et d'affections rénales. Par contre, les décès à domicile sont moins fréquents en cas de méningite $(38,5 \%$ des décès surviennent à domicile), de maladies infectieuses (43\%), de troubles nutritionnels et endocriniens $(43,3 \%)$, de maladies de l'appareil circulatoire $^{2}(43,8 \%)$ et de septicémie $(45,5 \%)$. En ce qui concerne le paludisme, première cause de décès, $53 \%$ des décès sont survenus à domicile, soit une proportion légèrement en dessous de la moyenne pour l'ensemble des causes (55\%). Selon les résultats des analyses, il n'y a pas d'association statistiquement significative entre le lieu de décès et le sexe, l'année de décès et le mois de décès.

\section{Analyses multivariées}

Le tableau 3 présente les résultats de modèles multiniveaux de régression logistique à intercept aléatoire du lieu de décès. Dans le modèle complet, il ressort que le fait de décéder à domicile est principalement influencé par l'âge, le milieu de résidence et la cause de la maladie. Les résultats montrent que les personnes âgées $(50$ et + ) sont significativement plus susceptibles à décéder à domicile (odds ratio $(O R)=12,9$, intervalle de confiance à $95 \%$ (IC) : I,93-87,25) que les personnes de moins de 14 ans; les personnes vivant en milieu rural ont presque six fois de risque de décéder à domicile que les personnes vivant en milieu urbain $(\mathrm{OR}=5.57$, IC 95\%: I,57-19,75). En retenant comme catégorie de référence l'ensemble des maladies infectieuses qui ont pu être identifiées (en excluant le paludisme), il ressort que les personnes décédées de maladies de l'appareil circulatoire, des autres maladies infectieuses et de septicémie sont significativement moins susceptibles de décéder à domicile. Les résultats multivariés confirment l'effet significatif du quintile de niveau de vie sur le fait de décéder à domicile ou dans un centre de santé : comparativement au quintile socioéconomique inférieur, les personnes des ménages de quintile socioéconomique supérieur sont moins susceptibles de décéder à domicile $(O R=0,18$, IC $95 \%(0,03-$ $0,95)$. Nous nous attendions à une association significative entre la distance séparant les ménages des centres de santé et le fait de décéder à domicile ou dans un centre de santé. Cela n'est pas le cas, après contrôle de toutes les variables, vraisemblablement parce que nous travaillons sur un territoire relativement limité en taille.

Dans le modèle vide, le niveau «village» a contribué à $10 \%$ de la variance et après inclusion des variables individuelles et environnementaux, et cet effet se réduit à $2,3 \%$. Ceci suggère que ces variables expliquent environ deux tiers de la variabilité intervillage dans les risques de décéder à domicile.

\footnotetext{
${ }^{2}$ L'hypertension tension artérielle représente environ $60 \%$ (29 sur 48) des cas de décès de causes liées aux maladies de l'appareil circulatoire.
} 
Tableau 3 : Estimation des odds ratios (et de l'intervalle de confiance de $95 \%$ ) du modèle de régression logistique binaire sur le fait d'être décédé à domicile

\begin{tabular}{|c|c|c|c|c|c|}
\hline \multirow[t]{2}{*}{ Variables } & & \multicolumn{2}{|l|}{ Modèle 0} & \multicolumn{2}{|l|}{ Modèle 4} \\
\hline & & Odd-ratio & IC (95\%) & Odd-ratio & IC (95\%) \\
\hline \multirow{2}{*}{ Sexe } & Masculin & & & 1 & \\
\hline & Féminin & & & 1,2 & $0,50-2,09$ \\
\hline \multirow{3}{*}{ Age } & $0-14$ & & & 1 & \\
\hline & $15-59$ & & & 3,54 & $I, 02-12,3$ \\
\hline & $60+$ & & & $25,2 * * * *$ & $3,7-87,25$ \\
\hline \multirow{2}{*}{ Avoir été à l'école } & Oui & & & 1 & \\
\hline & Non & & & 0,69 & $0,25-I, 90$ \\
\hline \multirow{3}{*}{ Statut matrimonial } & Célibataire & & & 1 & \\
\hline & Marié & & & 1,22 & $0,38-3,90$ \\
\hline & Séparé/divorcé & & & 2,69 & $0,52-|3,9|$ \\
\hline \multirow{2}{*}{ Profession } & Agriculteurs & & & 1 & \\
\hline & Non agriculteurs & & & 0,41 & $0,16-I, 03$ \\
\hline \multirow{2}{*}{ Ethnie } & Mossi & & & 1 & \\
\hline & Autre & & & 0,88 & $0,29-2,64$ \\
\hline \multirow{3}{*}{ Religion } & Musulmane & & & 1 & \\
\hline & Catholique & & & 0,98 & $0,42-2,28$ \\
\hline & Autre & & & 0,7 & $0,19-2,61$ \\
\hline \multirow{2}{*}{ Milieu de résidence } & Urbain & & & 1 & \\
\hline & Rural & & & $5.57 * * *$ & $1,57-19,75$ \\
\hline \multirow{5}{*}{ Quintile de niveau de vie } & QI (Très pauvre) & & & 1 & \\
\hline & Q2 & & & 0,64 & $0,2 I-I, 9 I$ \\
\hline & Q3 & & & 0,54 & $0,18-I, 64$ \\
\hline & Q4 & & & 0,37 & $0,11-1,15$ \\
\hline & Q5 (très nantis) & & & $0,22 * * *$ & $0,05-0,95$ \\
\hline \multirow[t]{3}{*}{ Distance par rapport } & $<5 \mathrm{~km}$ & & & 1 & \\
\hline & $5-9 \mathrm{~km}$ & & & 1,84 & $0,58-5,81$ \\
\hline & $10 \&+\mathrm{km}$ & & & 5,2 & $0,36-75, I$ \\
\hline \multirow{15}{*}{ Cause de décès } & $\begin{array}{llll}\begin{array}{l}\text { Maladies infectieuses } \\
\text { paludisme) }\end{array} & \text { (sans } & \text { le }\end{array}$ & & & 1 & \\
\hline & Tumeurs & & & 0,18 & $0,02-I, 57$ \\
\hline & Troubles nutritionnels et endocriniens & & & 0,12 & $0,01-I, 24$ \\
\hline & Maladies de l'appareil circulatoire & & & $0,07 * *$ & $0,01-0,68$ \\
\hline & Affections du tube digestif & & & 0,23 & $0,07-2,02$ \\
\hline & Affections rénales et urologiques & & & 0,24 & $0,02-2,67$ \\
\hline & Causes périnatales de décès & & & 0,16 & $0,023-1,15$ \\
\hline & Causes externes & & & 0,71 & $0,09-5,78$ \\
\hline & Indéterminé & & & 0,35 & $0,05-2,59$ \\
\hline & Broncho pneumo/pneumopathie & & & 0,28 & $0,04-2,19$ \\
\hline & Diarrhée présumée infectieuse & & & 0,3 & $0,03-2,65$ \\
\hline & Maladies infectieuses non précise & & & $0,11 * * *$ & $0,01-0,91$ \\
\hline & Méningite & & & 0,17 & $0,02-I, 78$ \\
\hline & Paludisme & & & 0,35 & $0,06-2,19$ \\
\hline & Septicémie & & & $0,05 * * *$ & $0,003-0,68$ \\
\hline Constante & & 1,79 & $1 ; 3,2$ & 0,29 & 0,$03 ; 3,28$ \\
\hline \multicolumn{6}{|l|}{ Random-effects } \\
\hline Village/secteur & & 1 & 0,$5 ; 0,9$ & 0,42 & $0,10-1,73$ \\
\hline Ménage & & 2,2 & 1,$1 ; 4,4$ & 2,08 & $0,89-4,84$ \\
\hline \multirow{2}{*}{$\begin{array}{l}\text { Conditional intraclass } \\
\text { correlation }\end{array}$} & Village & $10 \%$ & & $2,3 \%$ & \\
\hline & Ménage/village & $64 \%$ & & $57,7 \%$ & \\
\hline Log likelihood & & $-394,54 * * *$ & & $-342,44 * * * *$ & \\
\hline
\end{tabular}

\section{Discussion}

La proportion des décès qui ont lieu à domicile s'élève à $56 \%$ dans notre étude à Kaya, dans une ville moyenne. Cette proportion est inférieure à celle observée à Nouna (75\%), à l'Ouest du Burkina sur un site de surveillance démographique plus rural (Yé et al., 20ll). Cette forte proportion de décès qui survient encore à domicile dans une population qui habite dans une ville secondaire ou à ses abords 
montre que l'accessibilité financière et la capacité d'accueil des centres de santé seraient une barrière le recours aux soins.

Selon les résultats de l'analyse quantitative, l'âge est la seule variable individuelle significativement associée au lieu de décès (avec des personnes âgées plus susceptibles de décéder à domicile). Des études précédents ont déjà montré que le lieu du décès varie sensiblement avec l'âge (Anteneh et al. 2013; Gomes and Higginson 2006). Nous n'avons pas trouvé d'association significative entre le lieu de décès et les autres variables au niveau individuel tels que le sexe, la profession, l'ethnie, la religion. L'analyse descriptive avait mis en évidence une association avec l'instruction mais cette variable perd sa significativité après contrôle des autres variables individuelles.

Le milieu de résidence, le statut socioéconomique du ménage sont significativement associés à la probabilité de décéder à domicile. Ces variables figurent parmi les facteurs que Gomes et al., (Gomes and Higginson 2006; Daniel Munday et al. 2007) identifient comme les principaux déterminants du lieu du décès. Ces résultats reflètent les disparités d'accès aux soins. Selon Haddad et al.,(2004), les plus pauvres ont trois fois moins de chances de recourir aux services d'un professionnel de santé public ou privé pendant leur épisode de maladie. II est probable que les ménages les plus pauvres préfèrent ne pas "gaspiller » leurs maigres revenus pour un malade dont les chances de survie sont estimées peu élevées.

Notre recherche met également en évidence l'importance variation dans l'occurrence des décès à domicile selon les causes de décès. Environ 7 décès sur 10 de personnes ayant souffert de causes externes (accidents de transports/chutes), de causes indéterminées, de bronchopneumopathie/pneumopathie et d'affections rénales et urologiques ont lieu hors d'un centre de santé. La grande majorité des personnes décédées de broncho-pneumopathies/pneumopathies sont des personnes âgées (50 ans plus) qui sont déjà plus enclines à décéder à domicile. Dans la zone de Kaya, les insuffisances rénales chroniques causent de nombreux décès, et la plupart de ces derniers surviennent à domicile. Des travaux réalisés au Burkina Faso par Lengani et al. (Lengani et al. 1997, 2010) ont mis en évidence le faible recours aux soins de santé pour les malades souffrant d'insuffisance rénale. D'une part, deux tiers des malades souffrant d'insuffisance rénale chroniques se rendent au centre de santé trop tard, lorsqu'ils ont atteint une phase terminale. D'autre part, du fait du coût élevé de la prise en charge des insuffisances rénales dans les centres hospitaliers, les malades ont davantage recours à la médicine traditionnelle pour les pathologies des reins (Lengani et al. 20l0).

La majorité des décès par maladies infectieuses et parasitaires (autres que le paludisme) (64\%), de tumeur $(60,5 \%)$ et de diarrhée d'origine infectieuse (60\%) se produisent aussi à domicile.

En particulier, 10 décès causés par le $\mathrm{VIH} /$ sida ont pu être identifiés, mais seuls deux de ces décès sont survenus dans un centre de santé. Anteneh et al., (20/3) ont également mis en évidence cette propension des malades du sida à décéder à domicile en Éthiopie. Des études menées au Burkina Faso ont montré que la stigmatisation des personnes vivant avec le $\mathrm{VIH}$ et de leur entourage est un facteur qui amène ces malades à se priver de soins, surtout à un stage avancé de leur maladie (Bila and Egrot 2009; Gausset et al. 2012).

Enfin, les décès par suite de maladies de l'appareil circulatoires (notamment l'hypertension artérielle) et les cas de décès par suite de méningite se sont produits plus souvent dans un centre de santé. Les maladies cardiovasculaires connaissent un développement important au Burkina Faso. La prévalence de l'hypertension artérielle était estimée à $29,6 \%$ en milieu urbain en 2010 (Ministere de la santé, 20/4). Selon l'étude de Doulougou et al. (2014), la prévalence de l'hypertension artérielle s'élève à $10,5 \%$ dans la ville de Kaya (ville moyenne) et à 9,5\% en milieu rural à proximité de Kaya. Le fait que les décès causés par les maladies cardiovasculaires soient davantage concentrés dans les structures de santé signifie que les statistiques hospitalières vont surreprésenter cette cause de décès.

\section{Conclusion}

Cet article fait un premier pas vers une meilleure compréhension des facteurs associés au lieu de décès en Afrique sub-saharienne, un domaine de recherche qui commence tout juste à être exploré. La probabilité de décéder à domicile plutôt qu'au sein d'une structure de santé varie sensiblement avec le milieu de résidence et le statut socio-économique du ménage, tandis que les caractéristiques individuelles exercent une influence moindre (à l'exception de l'âge). La forte proportion des décès évitables qui surviennent à domicile malgré les efforts consentis pour rendre accessibles les services de soins interpelle les décideurs du système de santé et invite à mener plus d'actions visant à adapter l'offre des soins aux besoins des populations.

Vu le caractère exploratoire de notre étude basée sur des données d'enquête populationnelle, des recherches à une échelle plus large seraient nécessaires pour mieux comprendre les facteurs 
culturels, les croyances et les perceptions des populations qui conditionnent le lieu du décès. Aussi, dans un contexte où plus de la moitié des décès (plus de $55 \%$ ) se produisent hors des centres de santé, une enquête nationale par autopsies verbales sur un échantillon représentatif national des décès serait nécessaire pour établir les causes des décès afin d'élaborer des politiques vivant en renforcer le système de santé. De telles expériences ont été menées avec succès en Inde (Aleksandrowicz et al. 20|4), en Turquie (Akgün et al. 20I2) et en Zambie (Mudenda et al. 20 I I) notamment. L'organisation du prochain recensement au Burkina Faso (prévu en 2016) offre une opportunité unique de disposer d'informations détaillées sur les lieux du décès et leurs causes à l'échelle nationale.

\section{Remerciements:}

Aristide Romaric Bado a bénéficié d'un soutien financier du Programme Teasdale-Corti de I'Université de Montréal/Centre de recherche du CHUM) et de l'Unité de santé internationale pour cette recherche.

\section{Références bibliographiques}

Ahishakiyé, H. (2008). Dépenses de santé catastrophiques: cas du Burundi.

Akgün, S., Çolak, M., \& Bakar, C. (20I2). Identifying and verifying causes of death in Turkey: National verbal autopsy survey. Public Health.

Aleksandrowicz, L., Malhotra, V., Dikshit, R., Gupta, P. C., Kumar, R., Sheth, J., et al. (20|4). Performance criteria for verbal autopsy-based systems to estimate national causes of death: development and application to the Indian Million Death Study. BMC Medicine 20/4, 12.

Ali, T. S., Krantz, G., Gul, R., Asad, N., Johansson, E., \& Mogren, I. (20II). Gender roles and their influence on life prospects for women in urban Karachi, Pakistan: a qualitative study. Global health action, 4, 7448.

Anteneh, A., Araya, T., \& Misganaw, A. (20/3). Factors associated with place of death in Addis Ababa, Ethiopia. BMC palliative care, 12(I), 14.

Baider, L., \& Surbone, A. (2007). Patients' choices of the place of their death: a complex, culturally and socially charged issue. Onkologie, 30(3), 94-5.

Beccaro, M., Costantini, M., Giorgi Rossi, P., Miccinesi, G., Grimaldi, M., \& Bruzzi, P. (2006). Actual and preferred place of death of cancer patients. Results from the Italian survey of the dying of cancer (ISDOC). Journal of epidemiology and community health, 60, 4I2-4I6.

Beng, A., Fong, C., Shum, E., \& Goh, C. (2009). Where the elderly die: The influence of socio- demographic factors and cause of death on people dying at home. Ann Acad Med, 38(8), 676-83.

Beninguisse, G., \& Fournier, P. (2003). L'accessibilité culturelle: une exigence de la qualité des services et soins obstétricaux en Afrique. Etude De La Population Africaine.

Bila, B., \& Egrot, M. (2009). Gender asymmetry in healthcare-facility attendance of people living with HIV/AIDS in Burkina Faso. Social Science and Medicine, 69(6), 854-86I.

Brazil, K., Bedard, M., \& Willison, K. (2002). Factors associated with home death for individuals who receive home support services: a retrospective cohort study. BMC palliative care, $I(I), 2$.

Cárdenas-Turanzas, M., Torres-Vigil, I., TovalínAhumada, H., \& Nates, J. L. (20II). Hospital versus home death: results from the Mexican Health and Aging Study. Journal of pain and symptom management, 4I(5), 880-92.

Chvetzoff, G., Garnier, M., Pérol, D., Devaux, Y., Lancry, L., Chvetzoff, R., et al. (2005). Factors predicting home death for terminally ill cancer patients receiving hospital-based home care: The lyon comprehensive cancer center experience. Journal of Pain and Symptom Management, 30(6), 528-535.

Cohen, J., Bilsen, J., \& Miccinesi, G. (2007). Using death certificate data to study place of death in 9 European countries: opportunities and weaknesses. BMC Public, 7, 283.

Cohen, J., Houttekier, D., Onwuteaka-Philipsen, B., Miccinesi, G., Addington-Hall, J., Kaasa, S., et al. (20I0). Which patients with cancer die at home? A study of six European countries using death certificate data. Journal of Clinical Oncology, 28(I3), 2267-2273.

Costantini, M., Balzi, D., Garronec, E., Orlandini, C., Parodi, S., Vercelli, M., \& Bruzzi, P. (2000). Geographical variations of place of death among Italian communities suggest an inappropriate hospital use in the terminal phase of cancer disease. Public health, I/4, I5-20.

Craig, F., \& Mancini, A. (2012). Can we truly offer a choice of place of death in neonatal palliative care? Seminars in fetal \& neonatal medicine, 44, 6-II.

Doulougou, B., Kouanda, S., Bado, A., Nikièma, L., \& Zunzunegui, M. V. (20I4). Hypertension in the Adult Population of Kaya Health and Demographic Surveillance System in Burkina Faso: Prevalence and Associated Factors, 4(I), 94-I I0.

Gabrysch, S., \& Campbell, O. M. R. (2009). Still too far to walk: literature review of the determinants of delivery service use. BMC pregnancy and childbirth, 9, 34.

Gallo, W. T., Baker, M. J., \& Bradley, E. H. (200I). Factors associated with home versus institutional 
death among cancer patients in Connecticut. Journal of the American Geriatrics Society, 49(6), 77I-7.

Gausset, Q., Mogensen, H. O., Yameogo, W. M. E., Berthé, A., \& Konaté, B. (20I2). The ambivalence of stigma and the double-edged sword of HIV/AIDS intervention in Burkina Faso. Social Science and Medicine, 74(7), I037-1044.

Gomes, B., \& Higginson, I. J. (2006). Factors influencing death at home in terminally ill patients with cancer: systematic review. BMJ (Clinical research ed.), 332(7540), 5I5-2I.

Grunier, A., Mor, V., Weitzen, S., Truchil, R., Teno, J., \& ROY, J. (2007). Where people die: a multilevel approach to understanding influences on site of death in America. Medical care research and review: MCRR, 64(4), 35I-78.

Haddad, S. (2010). Protocole de recherche sur Implantation et impacts sur la santé et l'équité en santé du programme de la MEILUP, 15.

Higginson, I. J., Jarman, B., Astin, P., \& Dolan, S. (1999). Do social factors affect where patients die: An analysis of 10 years of cancer deaths in England. Journal of Public Health Medicine, 2I(I), 22-28. doi:10.1093/pubmed/2I.I.22

Ikezaki, S., \& Ikegami, N. (20II). Predictors of dying at home for patients receiving nursing services in Japan: A retrospective study comparing cancer and non-cancer deaths. BMC palliative care, $I O(\mathrm{I})$, 3.

Jack, B., \& O'Brien, M. (20I0). Dying at home: community nurses' views on the impact of informal carers on cancer patients' place of death. European journal of cancer care, 19(5), 636-42.

Klinkenberg, M., Visser, G., van Groenou, M. I. B., van der Wal, G., Deeg, D. J. H., \& Willems, D. L. (2005). The last 3 months of life: care, transitions and the place of death of older people. Health \& social care in the community, 13(5), 420-30.

Kouanda, S., Bado, A., Yaméogo, M., Nitièma, J., Gisèle, Y., Fadima, B., et al. (20I3). Profile: The Kaya HDSS, Burkina Faso: a platform for epidemiological studies and health programme evaluation. International Journal of Epidemiology, I-9.

Kouanda, S., Yaméogo, W. M. E., Ridde, V., Sombié, I., Baya, B., Bicaba, A., et al. (20l4). An exploratory analysis of the regionalization policy for the recruitment of health workers in Burkina Faso. Human Resources for Health, I2(Suppl I), S6.

Lazenby, J. M., \& Olshvevski, J. (20I2). Place of death among Botswana's oldest old. Omega, 65(3), I7387.

Lazenby, M., Ma, T., Moffat, H. J., Funk, M., Knobf, M. T., \& McCorkle, R. (20I0). Influences on place of death in Botswana. Palliative \& supportive care, 8(2), 177-85.

Lengani, A., Coulibaly, G., Laville, M., \& Zech, P. (1997). [Epidemiology of severe chronic renal insufficiency in Burkina Faso]. [French] Epidemiologie de l'insuffisance renale chronique severe au Burkina Faso. Cahier de Sante, 7(6), 379-383.

Lengani, A., Lompo, L. F., Guissou, I. P., \& Nikiema, J. B. (20/0). Medecine traditionnelle et maladies des reins au Burkina Faso. Nephrologie et Therapeutique, 6(I), 35-39.

McWhinney, I. R., Bass, M. J., \& Orr, V. (1995). Factors associated with location of death (home or hospital) of patients referred to a palliative care team. CMAJ: Canadian Medical Association journal = journal de l'Association medicale canadienne, I52(3), 36I-7.

McWhinney, I. R., Bass, M. J., Orr, V., Teno, J. M., Clarridge, B. R., Casey, V., et al. (2004). Family perspectives on end-of-life care at the last place of care. JAMA: the journal of the American Medical Association, 29I(3), 88-93.

Ministere de la sante. (20/4). Plan strategique integre de lutte contre les maladies non transmissible 2014-2018.

Mudenda, S. S., Kamocha, S., Mswia, R., Conkling, M., Sikanyiti, P., Potter, D., et al. (20ll). Feasibility of using a World Health Organizationstandard methodology for Sample Vital Registration with Verbal Autopsy (SAWY) to report leading causes of death in Zambia: results of a pilot in four provinces, 2010. Population health metrics.

Munday, D., Dale, J., \& Murray, S. (2007). Choice and place of death: individual preferences, uncertainty, and the availability of care. Journal of the Royal Society of Medicine, 100(5), 2II-5.

Munday, D., Petrova, M., \& Dale, J. (2009). Exploring preferences for place of death with terminally ill patients: qualitative study of experiences of general practitioners and community nurses in England. BMJ, 339(jul I 5 I), b239l-b239I.

Neergaard, M. a., Jensen, a. B., Sokolowski, I., Olesen, F., \& Vedsted, P. (20I2). Socioeconomic position and place of death of cancer patients. BMJ Supportive \& Palliative Care, I-8.

Ruiz-Ramos, M., \& Javier, G. (20I I). Place of death in Andalusia: influence of age, gender and cause of death. American Society of Clinical Oncology, III-II5.

Su, T. T., Kouyaté, B., \& Flessa, S. (2006). Catastrophic household expenditure for health care in a low-income society: a study from Nouna District, Burkina Faso. Bulletin of the World Health Organization, 84(I), 2I-7. 
Tang, S. T., Chen, C. C.-H., Tang, W.-R., \& Liu, T.W. (20I0). Determinants of patient-family caregiver congruence on preferred place of death in taiwan. Journal of pain and symptom management, 40(2), 235-45.

Wagstaff, A. (2002). Pauvreté et inégalités dans le secteur de la santé. Bull OMS. http://www.who.int/entity/bulletin/volumes//00to 108.pdf.

WHO. (2009). Normes d'autopsie verbale: établissement et attribution de la cause de décès.
Wright, A. A., Keating, N. L., Balboni, T. a, Matulonis, U. a, Block, S. D., \& Prigerson, H. G. (2010). Place of death: correlations with quality of life of patients with cancer and predictors of bereaved caregivers' mental health. Journal of Clinical Oncology, 28(29), 4457-64.

Xu, K., Evans, D. B., Kawabata, K., Zeramdini, R., Klavus, J., \& Murray, C. J. L. (2003). Household catastrophic health expenditure: a multicountry analysis. Lancet, 362(9378), I I I-7. 